\title{
BMJ Open Integrating the clinical pharmacist into the emergency department interdisciplinary team: a study protocol for a multicentre trial applying a non-randomised stepped- wedge study design
}

To cite: Vesela R, Elenjord $\mathrm{R}$, Lehnbom EC, et al. Integrating the clinical pharmacist into the emergency department interdisciplinary team: a study protocol for a multicentre trial applying a nonrandomised stepped-wedge study design. BMJ Open 2021;11:e049645. doi:10.1136/ bmjopen-2021-049645

- Prepublication history and additional supplemental material for this paper are available online. To view these files, please visit the journal online (http://dx.doi.org/10.1136/ bmjopen-2021-049645)

Received 03 February 2021 Accepted 25 October 2021

\section{A) Check for updates}

(C) Author(s) (or their employer(s)) 2021. Re-use permitted under CC BY-NC. No commercial re-use. See rights and permissions. Published by BMJ.

For numbered affiliations see end of article.

Correspondence to Dr Renata Vesela;

Renata.Vesela@sykehusapoteknord.no

\section{ABSTRACT}

Introduction The 'emergency department (ED) pharmacist' is an integrated part of the ED interdisciplinary team in many countries, which have shown to improve medication safety and reduce costs related to hospitalisations. In Norway, few EDs are equipped with ED pharmacists, and research describing effects on patients has not been conducted. The aim of this study is to investigate the impact of introducing clinical pharmacists to the interdisciplinary ED team. In this multicentre study, the intervention will be pragmatically implemented in the regular operation of three EDs in Northern Norway; Troms $\emptyset$, Bodø and Harstad. Clinical pharmacists will work as an integrated part of the ED team, providing pharmaceutical care services such as medication reconciliation, review and/or counselling. The primary endpoint is 'time in hospital during 30 days after admission to the ED', combining (1) time in ED, (2) time in hospital (if hospitalised) and (3) time in ED and/or hospital if re-hospitalised during 30 days after admission. Secondary endpoints include time to rehospitalisation, length of stay in ED and hospital and rehospitalisation and mortality rates.

Methods and analysis We will apply a nonrandomised stepped-wedge study design, where we in a staggered way implement the ED pharmacists in all three EDs after a 3, 6 and 9 months control period, respectively. We will include all patients going through the three EDs during the 12-month study period. Patient data will be collected retrospectively from national data registries, the hospital system and from patient records. Ethics and dissemination The Regional Committee for Medical and Health Research Ethics and Local Patient Protection Officers in all hospitals have approved the study. Patients will be informed about the ongoing study on a general basis with ads on posters and flyers.

Trial registration number NCT04722588.

\section{INTRODUCTION}

The main role of clinical pharmacists is to improve medication management to achieve the best possible health outcome for patients. More
Strengths and limitations of this study

The stepped-wedge design, recommended for complex interventions in healthcare (+).

- No spillover effect between study groups (+).

Inclusion of the total emergency department populations in all included hospitals (+).

- No specialised training of the interdisciplinary teams $(-)$.

Inclusion from only three hospitals in Norway (-)

specifically, clinical pharmacists work to optimise medication therapy, identify and prevent drug-related problems (DRPs), and consequently minimise the risk of medication errors. This is traditionally done by medication history taking, medication reconciliation (MedRec), medication review (MedRev) and medication counselling, but requires working directly with patients, physicians and other healthcare professionals and includes communication to ensure that medications are correctly used. ${ }^{1-6}$

The employment of clinical pharmacists in hospitals has shown improvement in many aspects of medicines safety, for example, prescribing appropriateness with reduction of potentially inappropriate medications from $17.0 \%$ to $12.2 \%$, reduction of potentially prescribing omissions from $2.2 \%$ to $0.7 \%^{7}$ and increased appropriate use of antimicrobials with almost $80 \%$ acceptance rate of pharmacist recommendations. ${ }^{8}$ Seven of twelve trials in a review by Kaboli et al reported on reduction of DRPs and medication errors. ${ }^{9}$ In fact, studies indicate that more than $80 \%$ of DRPs can be identified and solved with clinical pharmacist 
interventions. ${ }^{10} 11$ Studies also show reduction in hard and costly endpoints such as hospital utilisations,for example, in the study by Liu et al where hospitalisation rate was reduced from $32.5 \%$ to $22.2 \%$ when a clinical pharmacist was included in the interdisciplinary team. ${ }^{12}$

The inclusion of clinical pharmacists in emergency departments (EDs) has become standard in many countries and has led to a reduction in identified medication errors by $78 \%,{ }^{1314}$ reduced medication omissions and delay, ${ }^{15}$ 12-hour shorter hospital stays per patient, ${ }^{16}$ reduction in rehospitalisation by $5 \%,{ }^{17}$ and decreased mortality rates. ${ }^{18}$ There is a wide range of services provided by clinical pharmacists in the ED that has shown an effect in various countries and settings. ${ }^{19-21}$

In Norway, implementation of the clinical pharmacists in direct patient care has progressed slowly compared with countries such as the USA and the UK, and the majority of all hospital departments do not yet have access to clinical pharmacy services. ${ }^{22}{ }^{23}$ For the few clinical pharmacists working in Norwegian EDs, no standardised workflow or procedure has yet been established. In this study, we will investigate the impact of implementing ED pharmacists as part of the interdisciplinary team in three EDs in Northern Norway. The aim of this study is to explore the impact on length of stay (LOS), rehospitalisation and mortality.

\section{Hypothesis and objectives}

Our hypothesis is that the intervention will affect time in hospital during 30 days after admission to the ED, combining time in ED during stay, time in hospital during stay if hospitalised and time in ED and/or hospital if rehospitalised within 30 days after each ED admission. This, in turn, will reduce time before the first unplanned rehospitalisation, number of hospital re-admissions and mortality, which again may reduce healthcare costs.

\section{METHODS AND ANALYSIS}

This protocol is developed in accordance with the Standard Protocol Items: Recommendations for Interventional Trials (SPIRIT) 2013 statement (see online supplemental file 1 for SPIRIT 2013 checklist). ${ }^{24}$

\section{Study design}

The implementation of clinical pharmacists into the ED interdisciplinary team is a complex intervention where interactions between the pharmacists and the rest of the team will change how the overall service is provided in addition to the tasks that the pharmacists will introduce into the ED. The number and variability of outcomes also point at the complexity of the intervention. Therefore, there has been permitted a degree of flexibility and tailoring. The effect of the intervention will be assessed applying a non-randomised stepped-wedge trial design. ${ }^{25} \mathrm{~A}$ stepped-wedge design allows for the intervention to be rolled out sequentially, thus allowing to control for differences between study sites (vertical control) and longlasting impacts (horizontal control) during the study period. This is the gold standard when a conventional randomised controlled trial is not possible. ${ }^{2526}$

The intervention will be implemented in all three EDs over a 12-month period, starting with a 3-month control period in all EDs (planned start-up 1 February 2021). This period allows for baseline data collection before the intervention. After this period, we will consecutively roll out the intervention in 3-month intervals. Starting with the largest ED (Tromsø, 3 May 2021), continuing with the second largest (Bodø, 2 August 2021) and finally the smallest ED (Harstad, 1 November 2021), see figure 1, all EDs will have the intervention implemented during the last 3 months until the trial is terminated (planned 31 January 2022).

\section{Study settings}

This is a multicentre study including three EDs in Northern Norway Health Authority region; the University Hospital of North Norway (UNN) Tromsø, Nordland Hospital (NLSH) in Bodø and UNN Harstad with approximately 15000,12000 and 6000 patients presenting annually in the respective EDs. The three EDs operate similarly and receive patients who need immediate healthcare in case of acute illness or injury. Norway has a well-functioning primary care system, including municipal urgent care clinics providing ambulatory care outside of general practitioner (GP) office hours. In order to be admitted to the $\mathrm{ED}$, the patients need a referral either from GP or from a physician at an urgent care clinic. At the ED, the patient is met by an ED nurse and an ED physician (either an intern or a resident in specialty training), who perform the initial examinations and assessments of the patient. A senior physician is always on call in case of the need for a consultation. NLSH is the only ED with senior physicians situated in the ED during daytime. From the ED, patients are either admitted to a hospital ward, transferred to a municipally run health institution or discharged to their homes. Few EDs in Norway have pharmacists included in the interdisciplinary team, and many hospital wards do not have clinical pharmacist available.

\section{Study population}

All patients presenting to the EDs during the study period will be included in the study. Patients presenting during the

\begin{tabular}{|l|c|c|c|c|c|c|c|c|c|c|c|c|}
\hline Month & $\mathbf{1}$ & $\mathbf{2}$ & $\mathbf{3}$ & $\mathbf{4}$ & $\mathbf{5}$ & $\mathbf{6}$ & $\mathbf{7}$ & $\mathbf{8}$ & $\mathbf{9}$ & $\mathbf{1 0}$ & $\mathbf{1 1}$ & $\mathbf{1 2}$ \\
\hline Troms $\varnothing$ & $\mathrm{C}$ & $\mathrm{C}$ & $\mathrm{C}$ & $\mathrm{I}$ & $\mathrm{I}$ & $\mathrm{I}$ & $\mathrm{I}$ & $\mathrm{I}$ & $\mathrm{I}$ & $\mathrm{I}$ & $\mathrm{I}$ & $\mathrm{I}$ \\
\hline Bod $\varnothing$ & $\mathrm{C}$ & $\mathrm{C}$ & $\mathrm{C}$ & $\mathrm{C}$ & $\mathrm{C}$ & $\mathrm{C}$ & $\mathrm{I}$ & $\mathrm{I}$ & $\mathrm{I}$ & $\mathrm{I}$ & $\mathrm{I}$ & $\mathrm{I}$ \\
\hline Harstad & $\mathrm{C}$ & $\mathrm{C}$ & $\mathrm{C}$ & $\mathrm{C}$ & $\mathrm{C}$ & $\mathrm{C}$ & $\mathrm{C}$ & $\mathrm{C}$ & $\mathrm{C}$ & $\mathrm{I}$ & $\mathrm{I}$ & $\mathrm{I}$ \\
\hline
\end{tabular}

Figure 1 The stepped-wedge study design showing the distribution of control (C) and the intervention (I) periods during a 12-month study period. 


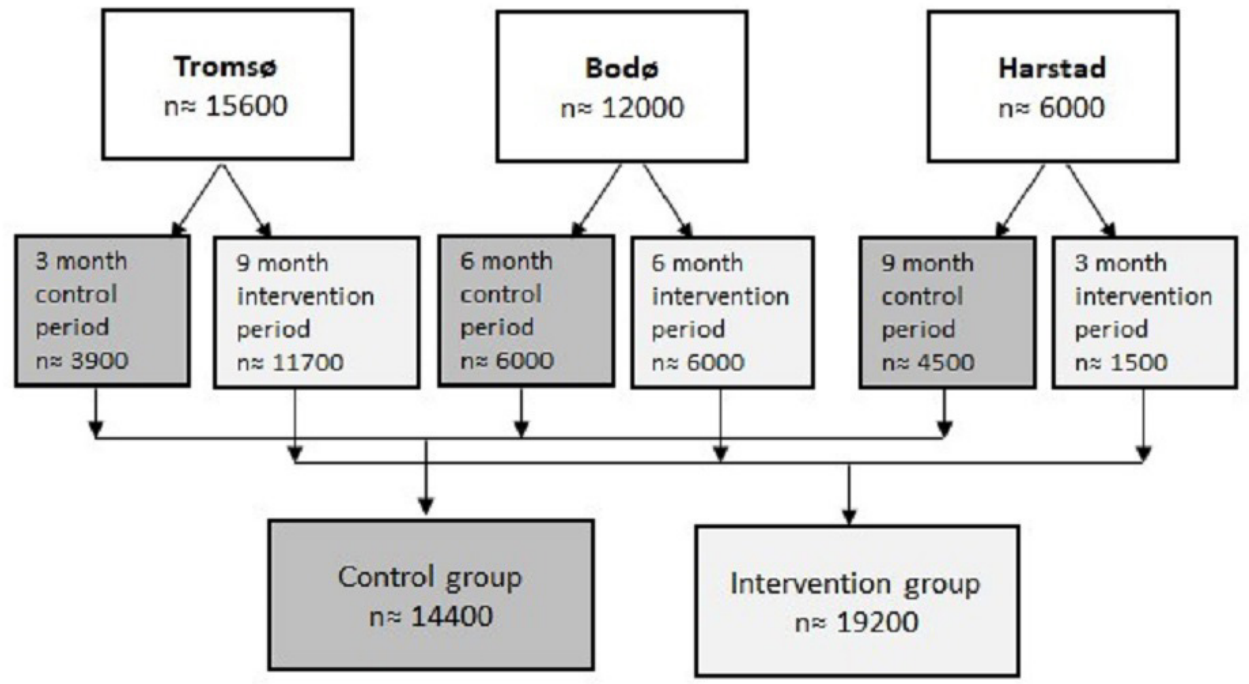

Figure 2 Flow chart of the anticipated population presenting to the emergency departments during the study period.

control period will be allocated to the control group $(n \approx 14$ 400), while patients presenting during the intervention period will be allocated to the intervention group $(n \approx 19$ 200), independently of whether they receive clinical pharmacist services or not, see figure 2. Patients, for whom data are not available retrospectively, will be excluded.

\section{Randomisation and blinding}

Neither EDs nor patients will be randomised. Randomising EDs would be preferable with the stepped-wedge design if a large number of EDs or equally sized EDs were included.

Neither staff nor patients will be blinded for the intervention, because it will be impossible to conceal the new member of staff. However, the ED pharmacists will be implemented as part of the daily-life work setting without announcing specifically to the patients that this is a new intervention.

\section{Standard care delivered during control periods}

The standard care procedures, which are similar in all three EDs, will be used in the control periods: Patients cared for in the EDs receive treatment from ED physicians and nurses, and no pharmacists are involved in any of the EDs. MedRec is usually performed by an intern or a resident in specialty training. The reconciled medication list is included in an admission note. The admission note is then uploaded to the electronic patient journal system that collects all patient medical data obtained in hospital. A standardised MedRev, by pharmacist standards, is not undertaken in the EDs. However, physicians may pause, change or add medications as appropriate. If the patient is admitted to hospital, the medications will be reviewed by physicians at the ward the proceeding day, where clinical pharmacists may be a part of the team.

On discharge, the patient's primary care physician (GP or institutional physician) receives a discharge summary. The discharge summary should include reasons for the hospitalisation, procedures and assessments made during admission and hospitalisation, and an updated medication list including a description of adjustments of medication therapy made during the hospital stay and recommendations for further follow-up. The primary care physician is responsible for follow-up of the patient and the patient's medication list after the hospital stay.

\section{The intervention delivered during intervention period}

During the intervention period, clinical pharmacists will be present in the EDs from 08:00 to 19:00 Monday to Friday. There will be two shifts, one shift is from 08:00 to $15: 30$ and another one is from 11.30 to 19.00 . Consequently, there will be clinical pharmacists available in the EDs during the hours of the day when the majority of patients arrive, and the pharmacist's capacity is doubled during the busiest time of the day. Early mornings are normally relatively slow paced and the pharmacists may use this time to follow-up on patients admitted during the night (from 19:00 to 08:00), in particularly those who have been admitted to wards without an assigned pharmacist.

The ED pharmacists will collaborate with the interdisciplinary teams and perform the following tasks according to patients' and EDs' needs: medication history taking, MedRec, MedRev, drug therapy recommendations, guidance on drug administration, medication information and counselling to patients/next of kin and healthcare personnel and communication about medications and changes in medication regimes, see figure 3. Standardised procedures, such as the integrated medicines management methodology, ${ }^{27}$ will be applied where possible. However, this is a complex intervention with a pragmatic approach where the intervention itself is not standardised, which better reflects the real-world setting. Inclusion of pharmacists in the team can lead to additional changes in the service when physicians and nurses use the pharmacists as a resource. Each patient will require different clinical interventions. ${ }^{28}$ Therefore, how, when and which task will be performed for each patient cannot be predetermined, but must be decided based on patient's needs and time constraints. Thus, not every patient will 


\section{EMERGENCY DEPARTMENT PATIENT FLOW}
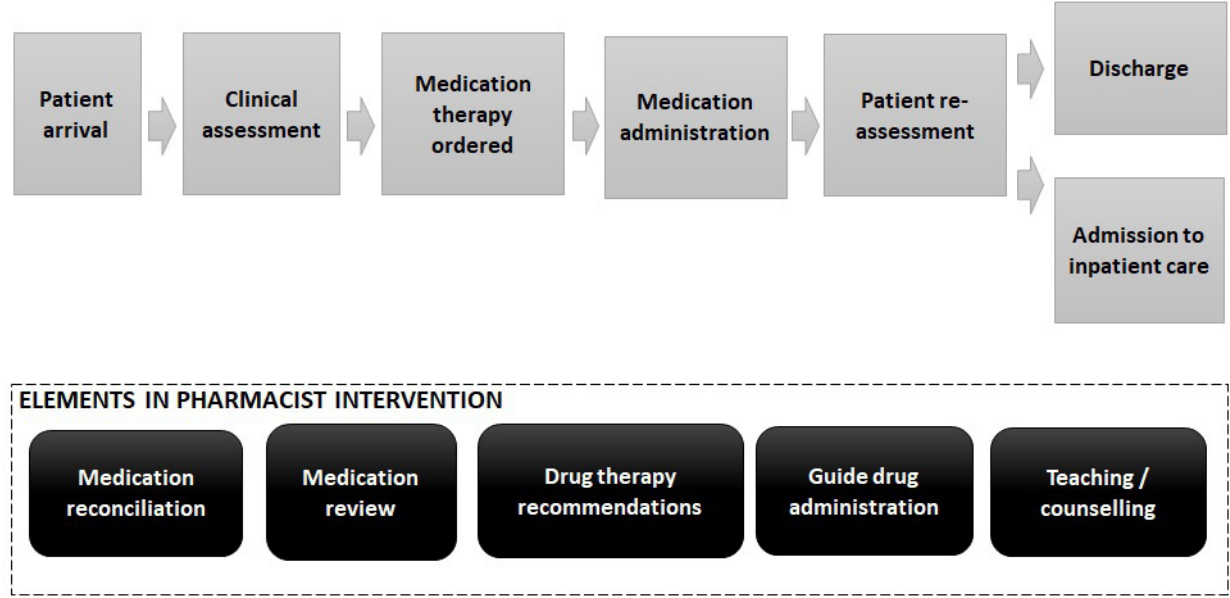

Figure 3 A pharmacist intervention in the emergency department (ED) put in the perspective of the ED patient flow.

receive the same intervention by the ED pharmacists, and not every nurse or physician would get discuss the same medication related issues with the ED pharmacists. The ED as a unit will be providing an extended service during the intervention period.

\section{Preparing for the intervention}

In order for physicians, nurses and pharmacists to prepare well for the intervention, we will introduce three initiatives that should ease the introduction of a new staff member; (1) information campaign to the EDs through emails, physical meetings and flyers, (2) theoretical and practical training of the clinical pharmacists in typical ED tasks in a fast-paced environment and (3) simulated ED team work with representative patient cases. The clinical pharmacists that are going to work in the EDs are trained as clinical pharmacists in other departments. In addition, they will go through a short training programme with lectures, seminars, discussions and observations, focusing on work flow in EDs and how the pharmacists may contribute.

\section{Patient and public involvement}

A patient representative has been involved throughout the whole duration of study planning period, already before application to funding was submitted. The one patient representative is member of a patient representative organisation where she, on a regular basis, discusses study-related issues with other patient representatives. More specifically, the patient representative is present at all project meetings where the whole project group is gathered to discuss study progress, design, research questions, outcome measures, patient inclusion and substudies (we are running substudies interviewing patients and healthcare personnel). We directly ask for advice on any aspects where patient perspectives are needed and she actively participates in discussions at all levels. As patients will not be asked for participation in this study, the patient representative has not been involved in patient recruitment. She is, however, involved in the patient information campaign and patient recruitment for the substudies. Except for scientifically result presentations, the study results will be disseminated to the study participants through public media, for example, newspaper articles or patient organisation presentations. The patient representative will play an important and active role in disseminating the results.

\section{Outcomes}

All outcomes below come from national registry data (the Norwegian Patient Registry and the cause of death registry).

\section{Primary outcome}

The primary outcome is 'time in hospital during 30 days after admission to the ED', which is a composite endpoint combining (1) time in ED during stay, (2) time in hospital during stay if hospitalised and (3) time in ED and/or hospital if rehospitalised within 30 days after each ED admission. This is an endpoint that has previously shown an effect in a Canadian study where pharmacist-led MedRev reduced time in hospital among high-risk patients under 80 years of age. ${ }^{16}$

Each patient can have more than one stay included in the study, but any admission during the 30-day time window after a previous admission will be excluded in order to avoid counting the stay twice, as an admission and a readmission in the previous stay. See figure 4 for a graphical representation of the inclusion and exclusion of stays.

\section{Secondary outcomes}

\section{Time to rehospitalisation (unplanned)}

We will measure time before the first unplanned rehospitalisation and compare the duration from the control period to the duration from the intervention period.

\section{0-day rate for rehospitalisation (unplanned)}

The 30-day rate for rehospitalisation during the control period will be compared with the trial period where ED pharmacists will be present in the ED. The rate will be measured by the number of patients who are rehospitalised within 30 days after their index stay. 


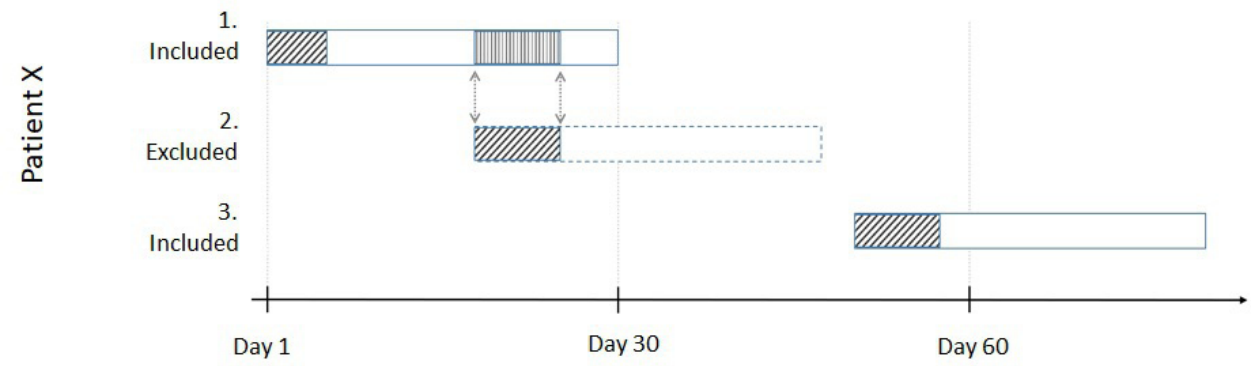

Figure 4 A graphical representation of the inclusion and exclusion of stays. Patient $X$ is admitted on day 1 and stays in the hospital for 5 days (first box). The patient then gets admitted again on day 18 (second box) for another 7 days. These 7-day count towards the primary endpoint during the 30-day time window after the first admission. However, to avoid double-counting time, the second admission is excluded as a separate stay. The third stay (third box) is an admission on day 49 and it is counted a new stay with its own 30 days.

\section{LOS in ED}

The ED LOS will be represented in minutes as discharge time from the ED (or time transferred to a hospital ward) minus admission time in the ED.

\section{LOS in hospital}

LOS in hospital will be calculated as discharge date minus admission date. ${ }^{29}$

\section{Mortality}

We will measure mortality rate during 30 days after admission to the ED.

\section{Sample size calculation}

The total number of admitted patients per month is about 1300, 1000 and 500 in Troms $\varnothing$, Bodø and Harstad, respectively. We assume that $20 \%$ will be missing complete registry data and will have to be excluded. This leaves us with 2240 admissions per month, 26680 admissions in total. Of these patients, we anticipate that 15360 admissions will occur during the intervention period.

Our primary outcome was previously applied in a Canadian study, where they showed a significant 0.5 -day reduction the primary endpoint after a similar intervention. ${ }^{16}$ If we assume a more conservative effect size of 0.25 days and a mean LOS in Norwegian hospitals of 4.2 days $(\mathrm{SD}=2)^{30}$ we can calculate the required sample size using adjusting a for stepped-wedge design. ${ }^{31}$ Using a significant level of $5 \%$ and power of $90 \%$ and an intraclass correlation of 0.001 (very little selection in who goes to the different EDs), we will need a minimum of 5222 admissions in each group.

\section{Data collection and follow-up}

We will collect data retrospectively from national health registries, patient records and hospital systems, see table 1. Study participants will be followed up for 3 months after each ED admission as described above. To adjust for long-lasting impacts, we will also collect data related to 6 months before and after each ED stay.

\section{Statistics and data analysis}

Data will be assessed for normality and analysed according to appropriate statistical distributions. The baseline demographic and clinical characteristics will be summarised using proportions, means and SD, or median and IQR, as appropriate. The reporting of results will follow the Consolidated Standards of Reporting Trials guidelines. ${ }^{32}$

Regression modelling will be used to adjust for potential confounders such as calendar time, this will be done using generalised estimating equations in order to accommodate the cluster nature of the data. Subgroup analyses based on variables such as age, gender and reason for visiting the ED will be done in order to study if any groups benefit more from our intervention. The main analysis will be done on all stays with an ED visit during the intervention time compared with all stays with a visit during the control period. The study statistician will be blinded to whether each individual patient visited the ED during the control or intervention period until the analysis is completed. All statistical tests will be interpreted with a significance level of $5 \%$ (two tailed).

Data from the study will also be used in other projects as described in discussion part.

\section{Table 1 Overview of variables to be collected on patient and pharmacist level}

\begin{tabular}{llll}
\hline Variable & Description & Data source & Timing/time interval \\
\hline $\begin{array}{l}\text { Demography and patient } \\
\text { information }\end{array}$ & $\begin{array}{l}\text { Year of birth, community, sex, place of stay, NPR } \\
\text { number, comorbidities }\end{array}$ & NPR & Retrospective \\
Stay in ED & EPJ & Retrospective \\
& $\begin{array}{l}\text { Hospital, triaging, time in, time out, site for discharge, } \\
\text { Mortality }\end{array}$ & NPR & 6 m. before and after ED visit* \\
& $\begin{array}{l}\text { Mortality within 30 days after ED index stay and cause } \\
\text { of death }\end{array}$ & NPJ & Retrospective \\
& CDR & 6 m. before and after ED visit* \\
\hline
\end{tabular}

*A larger period than the primary endpoint in order to adjust for long-lasing impacts in the analyses.

CDR, cause of death registry; ED, emergency department; EPJ, electronic patient journal; m, months; NPR, Norwegian Patient Registry. 


\section{Ethics and dissemination}

The study has been approved by the Patient Protection Officer at the Hospital Pharmacy of North Norway Trust and the three involved hospitals. The trial will be conducted in compliance with the protocol, the principles of Good Clinical Practice and the Declaration of Helsinki. Since our intervention will be implemented as a part of standard practice, patient consent will not be necessary. However, patients will be informed about the ongoing study on a general basis in all EDs with ads on TV screens, posters and flyers. Patients will have the opportunity to actively refrain from study participation, and information about how to do this will be easily available. The retrospective data collection from national registries has been approved by the Regional Committees for Medical and Health Research Ethics and local Patient Protective Officers at each hospital.

We aim to publish study results in international peerreviewed open access journals, at national and international conferences and in local, national and international media.

\section{DISCUSSION}

This intervention study is a part of an overarching project 'pharmacist in the ED' with an overall aim to investigate the impact of the ED pharmacist implementation on several aspects, not only patient safety outcomes. Consequently, a wide range of studies will be performed in addition to this intervention study, and data from the intervention study will also be applied to other studies. We will identify barriers for including the ED pharmacists and identify how the ED pharmacists should be working. We will apply interviews and observations in the EDs, to identify if the intervention will have an effect on primary care services. We plan to investigate if rate of visits to GPs are influenced. Also, we will investigate how medication regimes are influenced by the ED pharmacist intervention. Medication appropriateness will be determined through a systematic comparison of medication appropriateness in the intervention group compared with the control group. The medication appropriateness index is a possible tool. ${ }^{33}$ We want to identify which are specific pharmacy services and recommendations delivered by the ED pharmacists by applying journal data documented in the electronic patient journals (EPJ). The data on these interventions will be retrospectively collected from the EPJ and the interventions will be categorised into different activities (eg, MedRec, MedRev, patient counselling). The DRPs will be identified and outcomes after discussion with the interdisciplinary team registered. The clinical relevance of a randomly selected part of the interventions will be retrospectively evaluated by an expert team. We will explore the acceptance rate of pharmacist recommendations, which may be applied as a proxy for the clinical relevance of the recommendations made by ED pharmacists. We will also investigate whether the rehospitalisations in the study population are drug related. This may be done by applying expert groups and the Delphi methodology for agreement, or by applying the assessment tool for identifying Hospital Admission Related Medications $10 .{ }^{34}$ We aim to study whether the health-related quality of life (HRQoL) is influenced by the intervention. We will select a small and random part of the study population who will be asked to participate in an HRQoL study, where the EQ-5D VAS (Visual Analog Scale) tool will be applied. ${ }^{35}$ We will also investigate the cost-effectiveness of the intervention, a health economic simulation model evaluating the cost utility of the ED intervention will be developed. The simulation will compare future health of patients in two strategies; either with the ED pharmacists or with the current practice, with no pharmacists. Data from the other studies will be applied in the cost-effectiveness study.

This is the first study located in the literature testing a pragmatic real-world pharmacist approach, including all patients going through the ED throughout a whole year. Results will give valuable insight into outcomes of ED pharmacist involvement, and positive results may add speed to the implementation of pharmacists in ED settings world-wide. The main strength of the study is the stepped-wedge design, allowing for inclusion of the total population going through the ED in the study period. Another strength is the unbiased endpoint data collection from high-quality national registers. Some limitations do, however, exist, the main one being the inclusion of the pharmacists in the ED team. If they are not properly included, they may not be able to fully perform pharmacist services and consequently not able to influence patient care. Regarding generalisability, we believe results may have implications for both Norway, Scandinavia and other countries with a similar ED and hospital structure.

\section{Author affiliations}

${ }^{1}$ Hospital Pharmacy of North Norway Trust, Tromso, Norway

${ }^{2}$ Department of Pharmacy, UiT The Arctic University of Norway, Tromso, Norway ${ }^{3}$ Department of Health and Caring Sciences, Linnaeus University, Kalmar, Sweden

${ }^{4}$ Department of Medicine, Nordland Hospital Trust, Bodo, Norway

${ }^{5}$ Department of Community Medicine, UiT The Arctic University of Norway, Tromso, Norway

${ }^{6}$ Department of Public Health, The University of Copenhagen, Copenhagen, Denmark ${ }^{7}$ Department of Emergency Medicine, University Hospital of North Norway Trust, Tromso, Norway

${ }^{8}$ Department of Emergency Medicine, University Hospital of North Norway Trust, Harstad, Norway

\section{Twitter Eirik Hugaas Ofstad @eirikhugo}

Acknowledgements We are extremely grateful to all study participants, our patient representative Anne Lise Brygfjeld, ED employees, and our collaboration partners at UNN Harstad, UNN Tromsø, NLSH and the Hospital Pharmacy of North Norway Trust.

Contributors RE, RV, KS and BHG were involved in the study design. RE, RV, KS and BHG drafted the manuscript. ECL, EHO, TJ, BZ-H, TR, TW, LR, OMF, P-CV and HMF read and commented on the draft. All authors read and approved the final manuscript.

Funding This work is supported by the Northern Norway Regional Health Authority grant number HNF1483-19. The publication charges for this article have been funded by a grant from the publication fund of UiT-The Arctic University of Norway.

Disclaimer The sponsor has no part in collection, management, analysis and interpretation of the data, as well as writing and reporting study conclusions.

Competing interests None declared.

Patient consent for publication Not applicable.

Provenance and peer review Not commissioned; externally peer reviewed. 
Supplemental material This content has been supplied by the author(s). It has not been vetted by BMJ Publishing Group Limited (BMJ) and may not have been peer-reviewed. Any opinions or recommendations discussed are solely those of the author(s) and are not endorsed by BMJ. BMJ disclaims all liability and responsibility arising from any reliance placed on the content. Where the content includes any translated material, BMJ does not warrant the accuracy and reliability of the translations (including but not limited to local regulations, clinical guidelines, terminology, drug names and drug dosages), and is not responsible for any error and/or omissions arising from translation and adaptation or otherwise.

Open access This is an open access article distributed in accordance with the Creative Commons Attribution Non Commercial (CC BY-NC 4.0) license, which permits others to distribute, remix, adapt, build upon this work non-commercially, and license their derivative works on different terms, provided the original work is properly cited, appropriate credit is given, any changes made indicated, and the use is non-commercial. See: http://creativecommons.org/licenses/by-nc/4.0/.

\section{ORCID iDs}

Renata Vesela http://orcid.org/0000-0002-5417-8424

Renate Elenjord http://orcid.org/0000-0002-3384-6964

Elin C Lehnbom http://orcid.org/0000-0003-1428-5476

Eirik Hugaas Ofstad http://orcid.org/0000-0003-4821-6938

Torstein Risør http://orcid.org/0000-0002-2018-528x

Torbjørn Wisløff http://orcid.org/0000-0002-7539-082x

Kristian Svendsen http://orcid.org/0000-0003-3481-3539

Beate H Garcia http://orcid.org/0000-0002-0815-0383

\section{REFERENCES}

1 Viktil KK, Blix HS. The impact of clinical pharmacists on drug-related problems and clinical outcomes. Basic Clin Pharmacol Toxicol 2008:102:275-80.

2 Khalili H, Farsaei S, Rezaee H, et al. Role of clinical pharmacists' interventions in detection and prevention of medication errors in a medical ward. Int J Clin Pharm 2011;33:281-4.

3 Al-Hashar A, Al-Zakwani I, Eriksson T, et al. Impact of medication reconciliation and review and counselling, on adverse drug events and healthcare resource use. Int J Clin Pharm 2018;40:1154-64.

4 Chen P-Z, Wu C-C, Huang C-F. Clinical and economic impact of clinical pharmacist intervention in a hematology unit. J Oncol Pharm Pract 2020;26:866-72.

5 Hedegaard U, Kjeldsen LJ, Pottegård A, et al. Improving medication adherence in patients with hypertension: a randomized trial. $A m \mathrm{~J}$ Med 2015;128:1351-61.

6 Cabilan CJ, Boyde M, Currey E. The effectiveness of pharmacistled discharge medication counselling in the emergency department (explain): a pilot quasi-experimental study. Patient Educ Couns 2019;102:1157-63.

7 Ruiz-Millo O, Climente-Martí M, Navarro-Sanz JR. Improvement on prescribing appropriateness after implementing an interdisciplinary pharmacotherapy quality programme in a long-term care hospital. Eur J Hosp Pharm 2018;25:267-73.

8 Khdour MR, Hallak HO, Aldeyab MA, Nasif MA, et al. Impact of antimicrobial stewardship programme on hospitalized patients at the intensive care unit: a prospective audit and feedback study. $\mathrm{Br} \mathrm{J}$ Clin Pharmacol 2018;84:708-15.

9 Kaboli PJ, Hoth AB, McClimon BJ, et al. Clinical pharmacists and inpatient medical care: a systematic review. Arch Intern Med 2006;166:955-64.

10 Boșnak AS, Birand N, Diker Ömer, et al. The role of the pharmacist in the multidisciplinary approach to the prevention and resolution of drug-related problems in cancer chemotherapy. J Oncol Pharm Pract 2019;25:1312-20.

11 Sagita VA, Bahtiar A, Andrajati R. Evaluation of a clinical pharmacist intervention on clinical and drug-related problems among coronary heart disease inpatients: a pre-experimental prospective study at a general Hospital in Indonesia. Sultan Qaboos Univ Med J 2018;18:81-7.

12 Liu VC MI, Deol BB, Balarezo A, et al. Post-discharge medication reconciliation: reduction in readmissions in a geriatric primary care clinic. J Aging Health. 2018;898264318795571.

13 Stasiak P, Afilalo M, Castelino T, et al. Detection and correction of prescription errors by an emergency department pharmacy service. CJEM 2014;16:193-206.

14 Brown JN, Barnes CL, Beasley B, et al. Effect of pharmacists on medication errors in an emergency department. Am J Health Syst Pharm 2008;65:330-3.

15 Marconi GP, Claudius I. Impact of an emergency department pharmacy on medication omission and delay. Pediatr Emerg Care 2012;28:30-3.

16 Hohl CM, Partovi N, Ghement I, et al. Impact of early in-hospital medication review by clinical pharmacists on health services utilization. PLoS One 2017;12:e0170495.

17 Anderegg SV, Wilkinson ST, Couldry RJ, et al. Effects of a hospitalwide pharmacy practice model change on readmission and return to emergency department rates. Am J Health Syst Pharm 2014;71:1469-79.

18 Bond CA, Raehl CL. Clinical pharmacy services, pharmacy staffing, and hospital mortality rates. Pharmacotherapy 2007;27:481-93.

19 Morgan SR, Acquisto NM, Coralic Z, et al. Clinical pharmacy services in the emergency department. Am J Emerg Med 2018;36:1727-32.

20 Rothschild JM, Churchill W, Erickson A, et al. Medication errors recovered by emergency department pharmacists. Ann Emerg Med 2010;55:513-21.

21 Patanwala AE, Sanders AB, Thomas MC, et al. A prospective, multicenter study of pharmacist activities resulting in medication error interception in the emergency department. Ann Emerg Med 2012;59:369-73.

22 Rosmo K. Midtnorsk storsatsing på klinisk farmasi, 2021. Norsk Farmaceutisk Tidsskrift. Available: https://www.farmatid.no/artikler/ nyheter/midtnorsk-storsatsing-pa-klinisk-farmasi

23 Garcia BH, Halvorsen KH. Klinisk praksis - en veletablert undervisningsform I troms $\varnothing, 2020$. Norsk Farmaceutisk Tidsskrift. Available: https://www.farmatid.no/artikler/klinisk-praksis-enveletablert-undervisningsform-tromso [Accessed 07 Jan 2021].

24 Group TS. Spirit (standard protocol items: recommendations for interventional trials), 2021. Available: https://www.spirit-statement.org/

25 Hussey MA, Hughes JP. Design and analysis of stepped wedge cluster randomized trials. Contemp Clin Trials 2007;28:182-91.

26 Mdege ND, Man M-S, Taylor Nee Brown CA, et al. Systematic review of stepped wedge cluster randomized trials shows that design is particularly used to evaluate interventions during routine implementation. J Clin Epidemiol 2011;64:936-48.

27 Scullin C, Scott MG, Hogg A, et al. An innovative approach to integrated medicines management. J Eval Clin Pract 2007;13:781-8.

28 Craig P, Dieppe P, Macintyre S, et al. Developing and evaluating complex interventions: the new medical research council guidance. Int J Nurs Stud 2013;50:587-92.

29 Adlington K, Brown J, Ralph L, et al. Better care: reducing length of stay and bed occupancy on an older adult psychiatric ward. BMJ Open Qual 2018;7:e000149.

30 Statistisk sentralbyrå SN. Statistics Norway - official statistics about Norwegian society since 1876, 2020. Available: https://www.ssb.no/ helse/statistikker/pasient

31 Woertman W, de Hoop E, Moerbeek M, et al. Stepped wedge designs could reduce the required sample size in cluster randomized trials. J Clin Epidemiol 2013;66:752-8.

32 Hemming K, Taljaard M, McKenzie JE, et al. Reporting of stepped wedge cluster randomised trials: extension of the consort 2010 statement with explanation and elaboration. BMJ 2018;363:k1614.

33 Hanlon JT, Schmader KE, Samsa GP, et al. A method for assessing drug therapy appropriateness. J Clin Epidemiol 1992;45:1045-51.

34 Kempen TGH, Hedström M, Olsson H, et al. Assessment tool for hospital admissions related to medications: development and validation in older patients. Int J Clin Pharm 2019;41:198-206.

35 EuroQol. The EQ-5D-3L descriptive system, 2020. Available: https:// euroqol.org/ 